\title{
Premier Congrès de la Société Européenne d'Ophtalmologie
}

First Congress of the European Ophthalniological Society

Erster Kongreß der Europäischen Gesellschaft für Ophthalniologie

Athènes 1960

Rapports principaux - Main Lectures - Hauptreferate

Part I. Ophthalmologies, Vol. 142, No. 1 (1961)

Weekers, R. et Lavergne, G. (Liège):

Uvéites hypertensive et glaucomes post-iiiflammatoiress

Discussion: Francois, J. (Gand), Tzanidis, Th. (Salonique), Ver-

rey, F. (Zurich), Stankovic, I. (Beograd), Kalt, M. (Paris) ....

Huber, A. (Zurich): Das Glaukom hei komplizierter Heterochromie Fuchs

Discussion: Georgiades, G. (Salonique), IIollwich, F. (Jena), Ver-

rey, F. (Zurich), Trantas, N. C. (Athènes) 113

Jayle, G. E. et Ourgaud, A. G. (Marseille):

Les glaucomes post-opératoires 116

Discussion: Brissimis, N. (Volos), Lugossy, G. (Budapest), LeGrand, P. (Bruxelles), Sedan, J.

(Marseille), Kinnas, J. (Corinthe), Tzanidis, Th. (Salonique), Vassiliev, - (Sofia), Jayle, G. E.

(Marseille) 141

Part II. Ophthalmologica, Vol. 142, No. 2 (1961)

Miller, S. (London):

Glaucoma due to Lens Swelling 151

Discussion: Jayle, G. E. (Marseille) 159

Thiel, R. (Frankfurt a. M.):

Sekundärglaukom nach Epithel- oder Endotheleinwanderung in dieVorderkammer

Joannides, Th.; Karsourakis, N. und Velissaropoulos, P. (Athen):

Glaucoma capsulare 160

Discussion: Sampaolesi, R. (Buenos Aires), Joannides, Th. (Athen) 189

Francois, J. and De Rouck, A. (Ghent):

Electroretinography in Secondary Glaucoma 193

Karpe, G. and Wulfing, B. (Stockholm):

The ERG in Rapid Changes of Ocular Tension

Werner, L. (Dublin):

Glaucoma due to Haemorrhage in the Anterior or Posterior Segments 214

Discussion: Meyer-Schwickerath, G. (Essen) 226

Bietti, G. B. et Vanni, V. (Rome):

Glaucome secondaire à une obstruction veineuse extra-oculaire . . 227

Discussion: Moro, F. (Padua), Bietti, G. B. (Rome) 263

Vannas, S. (Helsinki):

Glaucoma due to Thrombosis of the Central Vein of the Retina . . 266 
Discussion: Bietti, G. B. (Rome), Tzanidis, Th. (Salonique), Moro, F. (Padoue), Weinstein, P. (Budapest), Santonastaso, A. (Padoue), Vannas, S. (Helsinki) 280

Heinc, A. (Olomous):

Glaucoma Traumaticum 283

Discussion: Sabbadini, D. (Milan), Wirth, A. (Rome), Vejdovsky,

V. (Olomouc) 300

Miron, M. S. (Bucarest):

Le glaucome par atrophie essentielle progressive de Гiris $\quad 301$

Discussion: Georgiades, G. (Salonique), BÉGUÉ, H. (Paris), Busch, K.-Th. (Leipzig), Zintz, R. (Freiburg i. Br.), Weinstein, P. (Budapest), Pouchkovskaja, N. (Odessa), Sedan, J. (Marseille), Miron, M. A. (Bucarest) 324

Moreu, A. (Santiago de Compostela):

Glaucoma secundario consecutivo a seclusion pupilar $\quad 330$

Discussion: Barraquer, J. (Barcelone), Strampelli, B. (Rome) . . 351

Ohrt, V. (Aarhus): Glaucoma due to Rubeosis Iridis Diabetica

Discussion: Francois, J. (Gand), Bruckner, R. (Basel), Tzanidis,

Th. (Salonique) 364

Böck, J. und Stepanik, J. (Wien):

Glaukom bei thyreogenem Exophthalmus 365

Discussion: Bietti, G. B. (Rome), Radnot, M. (Budapest), Huber, A. (Zurich), Tzanidis, Th. (Salonique), Rohrschneider, W. (Mün-chen), Böck, J. (Wien) 376

Papolczy, F. (Budapest):

Glaucoma due to Intraocular Tumours $\quad 379$

Discussion: Tzanidis, Th. (Salonique) 386

Part III. Communications

C. Ces travaux paraissent dans un volume supplémentaire à Ophthalmologica

Vol. 142 (1961) These papers are published in a supplement volume to Ophthalmologica

Vol. 142 (1961) Diese Arbeiten erscheinen in einem Supplementband zu Ophthalmologica

Vol. $142(1961)$ 